the amounts employed in his experiments. 2. Professor Hewlett knows very well that the "germicidal value" affords significant information only when several antiseptics are compared under precisely similar conditions. In our original communication we gave the comparative values, as obtained in repeated tests, from which the high potency of flavine (acriflavine) was clearly apparent. Now Professor Hewlett finds fault with our results in the case of flavine, but he does not accu e us of giving unjust figures with regard to the old antiseptics, and it is by the latter that the comparative potency of flavine is estimated. Further, in his own work on flavine he omits all comparisons; we feel sure that Professor Hewlett would not desire readers to believe that he wished them to accept his statements as mere dogmatic assertions and yet that is in effect what he does. The general medical reader, who is himself without laboratory facilities, can only draw conclusions from the data afforded, and Professor Hewlett omits half of the data which are necessary in order to support his contention that we have exaggerated the potency of flavine. Again, Professor Hewlett raises the old point that the action of an antiseptic when mixed with a quantity of pus affords an important indication as to its therapeutic efficiency in an infected wound. From the point of view of use as a test medium, pus is so extremely inconstant in composition that results vary enormously; but, as on a previous occasion, ${ }^{3}$ we can only repeat that any surgeon who treated infected wounds by mixing up antiseptic with the pus contained in them, would entertain a curious idea as to his functions !

It seems, however, that the real meaning of Professor Hewlett's work is an attempt to give expression to the view that a substance which is to possess any therapeutic value as an antiseptic must rapidly kill organisms. The time-hallowed tests employed in the standardising of antiseptics, of course, gave information on this point and almost on nothing else ; the practical inefficiency of the substances, which were accredited by such tests, e.g. phenol and mercuric chloride, when it came to putting an end to suppuration in infected wounds or to preventing its occurrence, is well known. Now, we have shown that one of the pre-eminent qualities of the flavine bodies is their antiseptio power in the true sense of the word, i.e., their capacity-in a serous medium, such as exists on the surface of a wound which is subjected to the ordinary procedures of surgical cleansing - for inhibiting the activity of organisms in very high dilutions, which are relatively harmless to the tissues. This combination of desirable properties in one substance is new ; but the idea of antiseptic action is not new; the conception of its value appears, however, to be novel to those who have concerned themselves with the cleansing of rubber gloves, sterilisation of infected rooms or sputum, and other common applications of antiseptics (purposes for which we have never recommended flavine); and, being novel, it seems to be either misunderstood or else regarded as odious according to the temperament of the critic. ${ }^{4}$ We shall, however, endeavour to pursue our search for those properties of antiseptics which can be determined in the laboratory and which are of use as indicating substances of therapeutic value, and we shall be content to be judged in the long run by the massed opinion of those who are bound to rely on such adjuncts in the treatment of their patients. We do, however, deprecate destructive criticism, which contributes little or nothing to advance towards the solution of a most urgent problem, but which almost seems devised-however unintentionally - in order to "scare off" those who might be inclined to give an unbiassed trial to substances for whose utility in practice there is already a great body of evidence.

I am, Sir, yours faithfully,

Middlesex Hospital, Oct. 11th, 1917. C. H. Browning.

\section{WAR DEAFNESSES.}

To the Editor of $\mathrm{TH} \mathrm{E} \quad \mathrm{AN} \mathrm{CET}$.

SrR,-In a leading article on War Deafnesses in your issue of Oct. 13th you refer to a paper of ours which appeared in THE LANCET of the previous week. Two criticisms call for reply. Firstly, you state your belief that " any other powerful stimulus applied during the removal of the subconscious determination against hearing would

3 Ibid., 1917, i., p. 177.
* See Browning, THE LANCET, Sept. 15th, 1917. have been as effective as suggestion proved," but you do not give any idea of the nature of such stimuli. Both of our cases had been treated without success for several month before we saw them by all kinds of powerful stimuli, and we applied every means we could devise before resorting to the pseudo-operation, the instantaneous and complete success of which surely justified the method. There is no question of its "frequent" use, as we have only applied it in the very exceptional cases in which all other treatment, such as simple re-education and persuasion, or suggestion with or withou the aid of electricity, whilst awake, asleep, in the hypnotic state, or under light etherisation, had failed.

Secondly, you express doubt as to the correctness of our conclusion that total deafness associated with continued excitability of the posterior labyrinth is evidence of hysterical deafness, because "two cases are quite an insufficient basis for such a generalisation." But our conclusion is based on over 200 cases, not on the two cases which we quoted in detail only because of the exceptional interest attached to the dramatic manner in which they were cured. Before our conclusion can be rejected some very much stronge evidence is required than a bald statement to the effect that " cases of mild labyrinthitis occur in which there appears to be total deafness, but in which the canal reflex can still be excited." What is the evidence for this ? Our experience teaches us that in such cases the mild labyrinthitis leads to mild deafness, the complete deafness being due to the addition of an hysterical element. This is a matter of extreme practical importance, as in such cases we have always succeeded in removing the hysterical element by suggestion so that partial hearing was noticed, sometimes many months after a diagnosis of permanent total deafness had been made else where. On the other hand, acceptance of the notion that mild labyrinthitis may lead to complete deafness must result in the neglect of such treatment and the consequent perpetuation of the condition. There can thus be no question that from the patient's point of view our conclusions and the results which follow from these are infinitely more desirable than the pessimistic view taken in your leading article. We are, Sir, yours faithfully,

\section{Netlej, Oct. 15th, 1917.}

ARTHUR F. HURST,

* * The success in the hands of Major Hurst and Captain Peters of the pseudo-operation as a sequel to a course of suggestion in the two cases cited is so striking that others will undoubtedly follow in their steps, and we therefore expressed the hope that some other stimulus less derogatory to the status of surgery might be found equally efficient. The existence of cases of total deafness with retention of canal excitability is a matter of pre-war experience.-ED. L.

\section{INSANE SAILORS AND SOLDIERS.}

\section{To the Eiditor of THE LANCET.}

SIR,-The Board of Control has issued a circular with reference to "Service patients," or, in other words, sailors and soldiers who have become insane or are otherwise mentally affected. To suggest, as has been done, that asylums would in any case have had to receive insane sailors and soldiers as paupers and keep them as such is introducing into the question the lowest argument of all-the pecuniary one. And the question is not yet and by no manner of means settled. At the present moment insane soldiers are being admitted to county asylums as pauper lunatics through the medium of the Poor-law.

I $\mathrm{wi} \cdot \mathrm{h}$ to comment on the Circular of Instructions issued by the "Board of Control" on June 27th of this year relating to the classification and treatment of insane sailors and soldiers as "Service patients." Do not let us, then, obscure the issues by wondering what we are to do with insane sailors and soldiers after the war and how to out their cost of maintenance to the lowest possible limit, which seems to be the end in view. The question of cost of both present and future accommodation and maintenance ought not to be approached in a parsimonious spirit, and yet it is at the root of the matter, because up to the present the Government has shown no desire to build asylums for the accommodation of insane sailors and soldiers, nor to provide for their accommodation other than in pauper asylums.

But the nation's indebtedness to those broken men calls for provision for them other than in pauper asylums and in 